\title{
Rapid and Sensitive Nano-Immunosensors for Botulinum
}

\section{Hui-Pin Chenga and Han-Sheng Chuang, ${ }^{a, b, *}$}

a Department of Biomedical Engineering, National Cheng Kung University, Taiwan

b Center for Micro/Nano Science and Technology, National Cheng Kung University, Taiwan
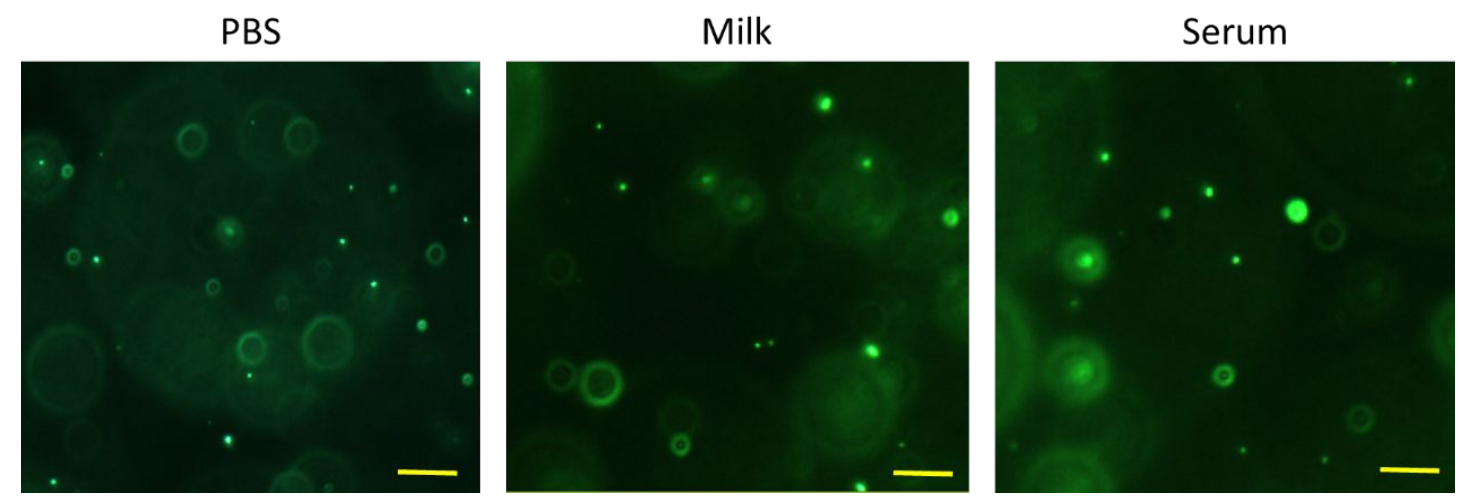

Figure S1 Representative images of 200-nm particles grafted with $80-\mathrm{nm}$ AuNPs in different sample matrices (from left to right: PBS, milk, and serum). The scale bar is $20 \mu \mathrm{m}$.

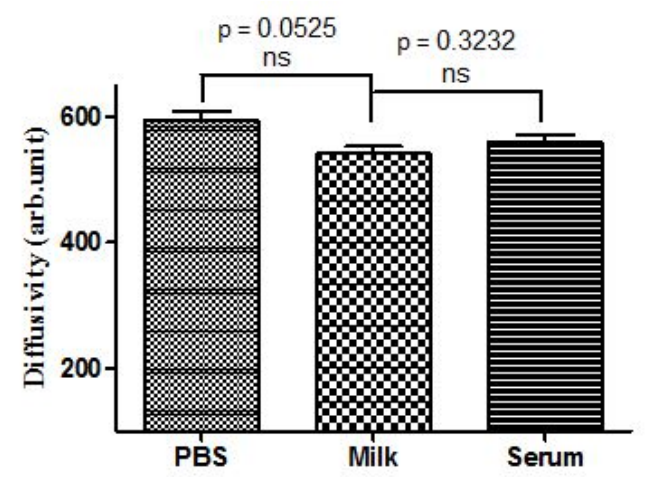

Figure S2 Diffusivity values of none conjugated AuNPs in three matrices. Statistical analysis with the Student $t$ test shows no significant differences between each other $(n=5)$. 